Thorax (1957), 12, 159.

\title{
THE SURGERY OF PRIMARY PULMONARY TUBERCULOSIS IN CHILDREN
}

\author{
BY \\ J. T. CHESTERMAN \\ Sheffield
}

(RECEIVED FOR PUBLICATION JANUARY 31, 1957)

The portal of entry in the great majority of cases of primary tuberculous infection is through the lungs. Fortunately, the resulting intrathoracic lesions are not of much clinical significance in many children, but this primary pulmonary infection may show itself as:
1. Pulmonary lesion
(a) Bronchopneumonia
(b) Cavitatary disease

2. Gross enlargement of

(a) Pressure

glands with complica-

(b) Infection

tions due to

(c) Fibrosis

3. Massive pleural effusion Rare

4. Disseminated lesions. . Local and diffuse

It is important to recognize that the illness of the patient does not bear any definite relationship to the size of the lesion, and, second, that the tuberculous process often burns itself out but leaves permanent damage to the lung which leads to nontuberculous pneumonitis and bronchiectasis.

Disseminated lesions will not be discussed. Massive pleural effusion is rare and occurs in about $5 \%$ of clinically infected children, but is usually benign until adolescence is reached (Bentley, Grzybowski, and Benjamin, 1954). In patients with little resistance a massive effusion may produce a fibrothorax necessitating a decortication operation within three months of onset in spite of repeated aspiration and antibiotic treatment. This rapid development of a fibrothorax appears to occur almost entirely in the coloured races.

Children suffering from pulmonary lesions are treated on the same general principles as are applicable to adults, though resection is the only form of surgery desirable when operative intervention is indicated, as it leaves practically no deformity. My first resection in this type of case (Hospital No. 41093) was a pneumonectomy for cavitatary tuberculosis of the left upper lobe and bronchiectasis of the left lower lobe performed on a boy of 5 years of age in 1943. He is now well and a manual worker (1957).

\section{Gross Glandular EnLargement}

The main surgical problem, however, is the gross enlargement of the glandular element of the primary complex. Gross enlargement has been defined by Bentley and others (1954) as a lesion occupying a greater space than that between the upper border of the seventh rib and the lower border of the eighth rib posteriorly on a standard postero-anterior chest film. He found gross enlargement in some $8 \%$ of 317 cases under observation for glandular enlargement. He also noted that gross enlargement occurred in $4 \%$ of cases under 5 years and $11 \%$ of cases over that age and that boys were affected twice as frequently as girls. Radiologically, the glandular enlargement reaches its maximum some two to six months after the date of the primary infection (Bentley and others, 1954).

The precise course of the glandular enlargement does not appear to be altered by the anti-tuberculosis drugs even if given in large doses over many months as in cases of tuberculous meningitis (Lorber, 1957). Such cases show a comparable course to untreated cases as far as the glandular enlargement is concerned.

The incidence of cases requiring surgical intervention, even in gross glandular enlargement, appears to be small. There are not any adequate statistics on the subject, but my impression is that it amounts to about $\frac{1}{2} \%$.

\section{SURGICAL INTERVENTION}

Surgical intervention is indicated in the following five conditions:

1. Acute perforation of a major air passage which causes severe respiratory embarrassment; the majority of such perforations do not cause a serious episode, but, if they do, immediate bronchoscopic aspiration is a live-saving measure.

CASE Note.-G. E., a girl, born on August 29, 1950 (Hospital No. 52/5262), was under observation for a primary tuberculous infection. Her mother had phthisis. 


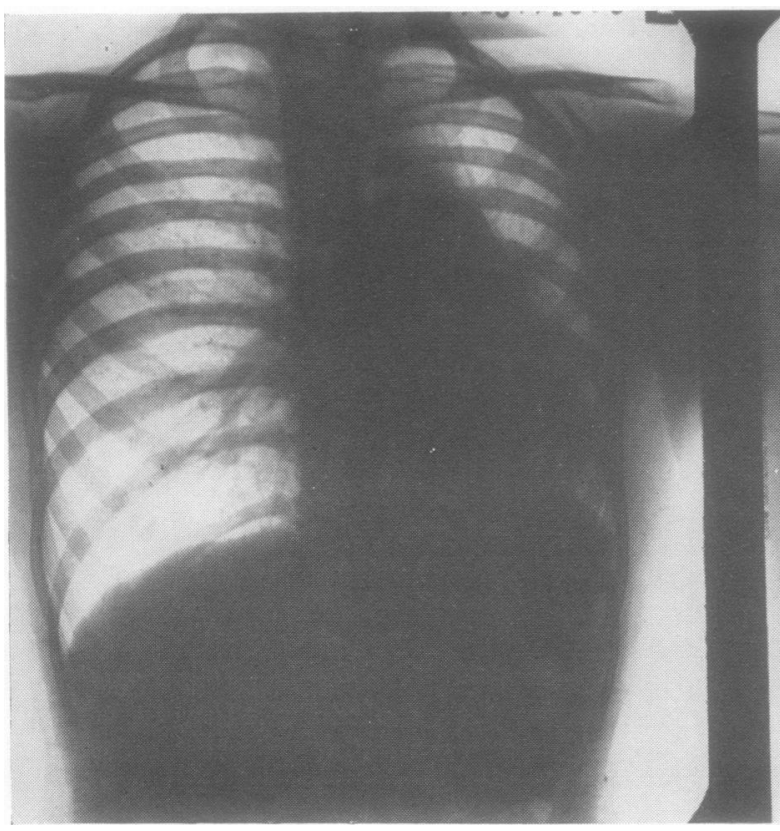

FIG. 1a.-Pre-operative film showing partial collapse of the left lung.

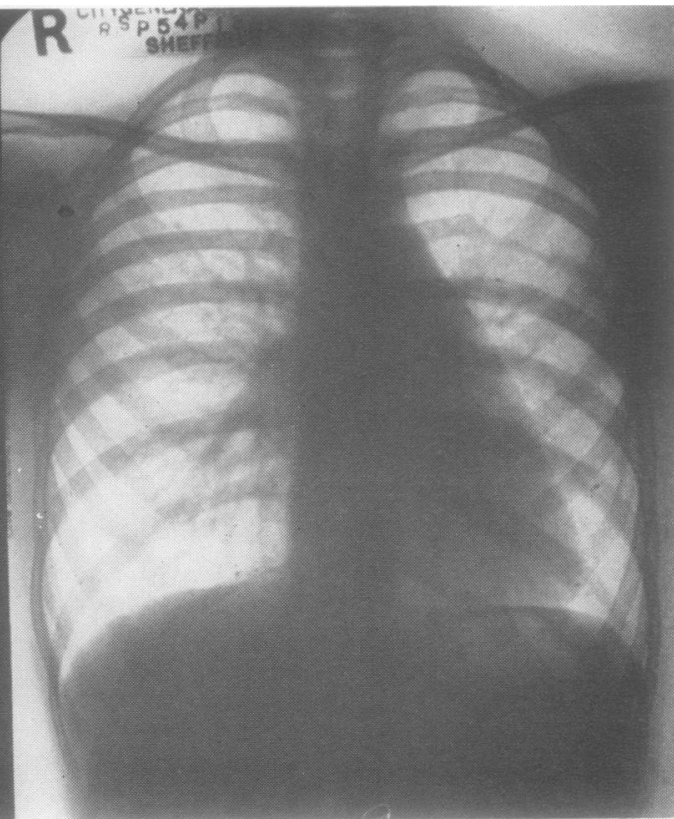

FIG. 1b.--Post-operative film showing complete expansion.
On June 10, 1952, the patient was suddenly taken with severe respiratory obstruction. Immediate bronchoscopy without anaesthetic was performed and $5 \mathrm{ml}$. of blood and caseous material was aspirated from the carina by the site of a perforation in the right postero-lateral wall of the trachea. A radiograph taken 12 hours later showed partial collapse of the right lung and a mediastinal mass. Her condition rapidly improved and a film taken $\overrightarrow{\overrightarrow{0}}$ three years later showed no evidence of disease.

2. The perforation of a glandular mass into a main airway which causes recurrent episodes of obstruction; this requires excision of the glands ando suture of the perforation.
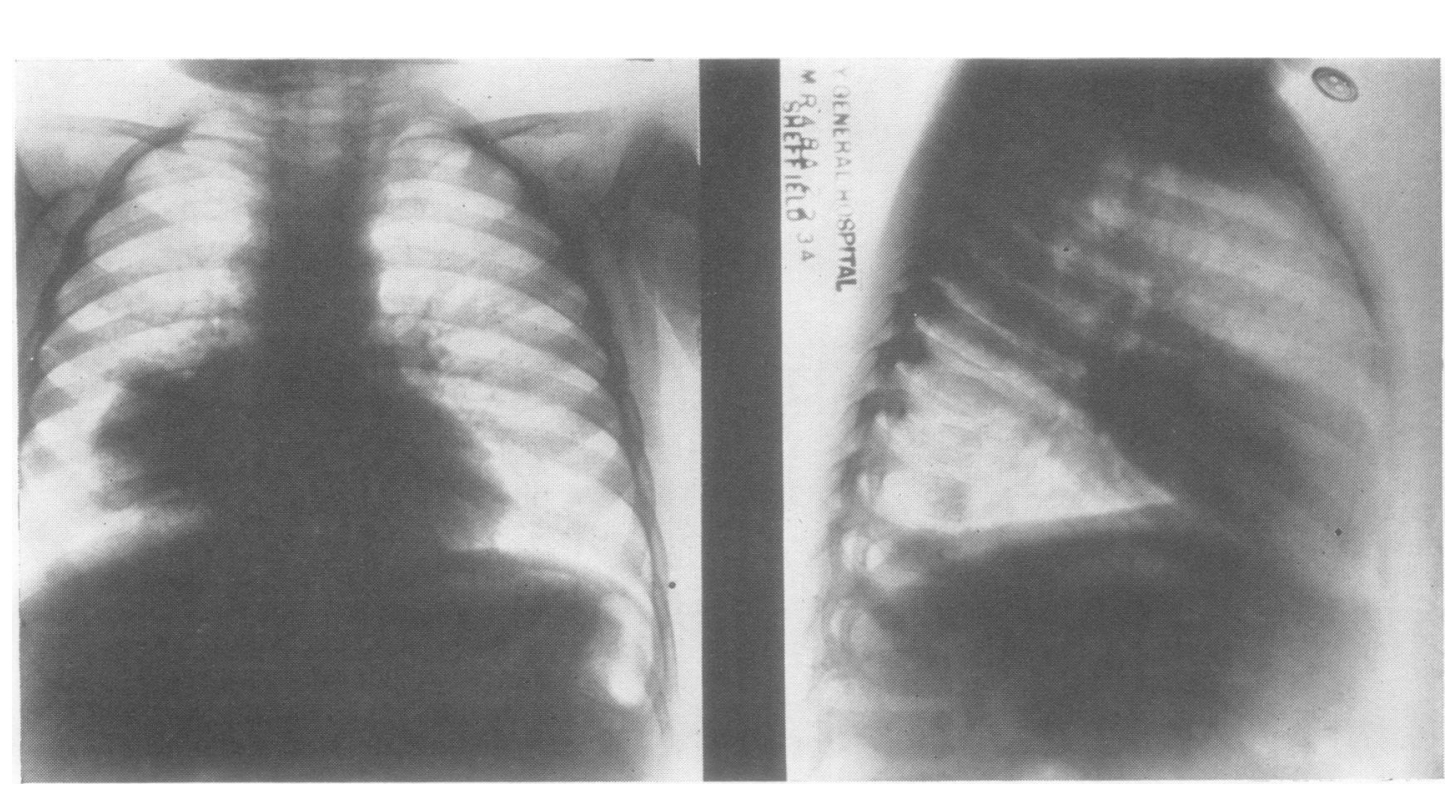

FIG. $2 a$ and $b$.-Films showing enlargement of glands which persisted for four months without change. 
CASE Note.-A. H., a girl, born on October 11, 1943 (Hospital No. 54/30934), was admitted with a history of "pneumonia" one month before, since when she had been short of breath, had a marked wheeze, and recurring attacks of partial collapse of the left lung (Fig. 1a). On February 17, 1954, bronchoscopy under general anaesthesia showed a complete block of the left lower lobe bronchus, and the obstruction was partially relieved. On February 25 bronchoscopy was repeated for recurrence of the obstruction. On March 20 further obstruction took place, so the mass of tuberculous glands was excised and the perforation sutured. All the gland showed marked caseation except the one causing the perforation which showed mainly an endothelial reaction without necrosis. Recovery was uneventful and she has remained well (1957) (Fig. 1b).

3. Glandular pressure which gives rise to bronchial obstruction and pulmonary collapse lasting more than one month; this requires excision of the mass, or, if this is impossible, its incision and removal of its contents. This is particularly true if the mass is situated in the right paratracheal region, as the incidence of haematogenous spread (Bentley and others, 1954), phthisis, residual non-tuberculous bronchiectasis, and indurative mediastinitis with superior vena caval obstruction is higher in this situation than elsewhere. Excision should not be delayed more than a few weeks, otherwise residual lesions involving the lung or mediastinum are apt to occur.
CASE Note.-T. W., a boy, born on December 12, 1945 (Hospital No. 54/8489), was admitted early in 1948 with a large glandular mass by the trachea and right hilum of the lung. A bronchogram also showed collapse of the anterior segment of the right upper lobe, the right middle lobe, and the medial basal segment of the right lower lobe. The lesion remained unchanged for three months (Figs. $2 a, 2 b, 3$ ). On May 10, 1948, the

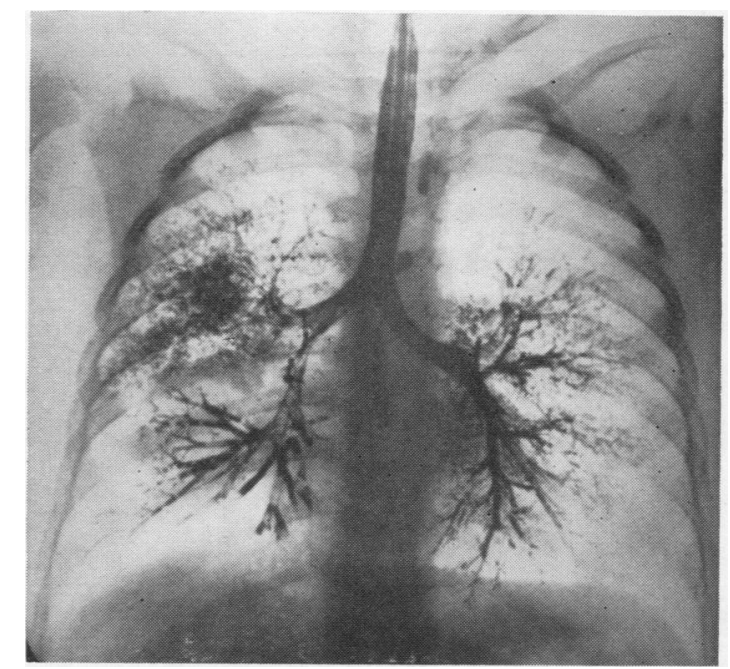

FIG. 3.-Pre-operative bronchogram showing the areas of collapse of the right lung due to glandular pressure.

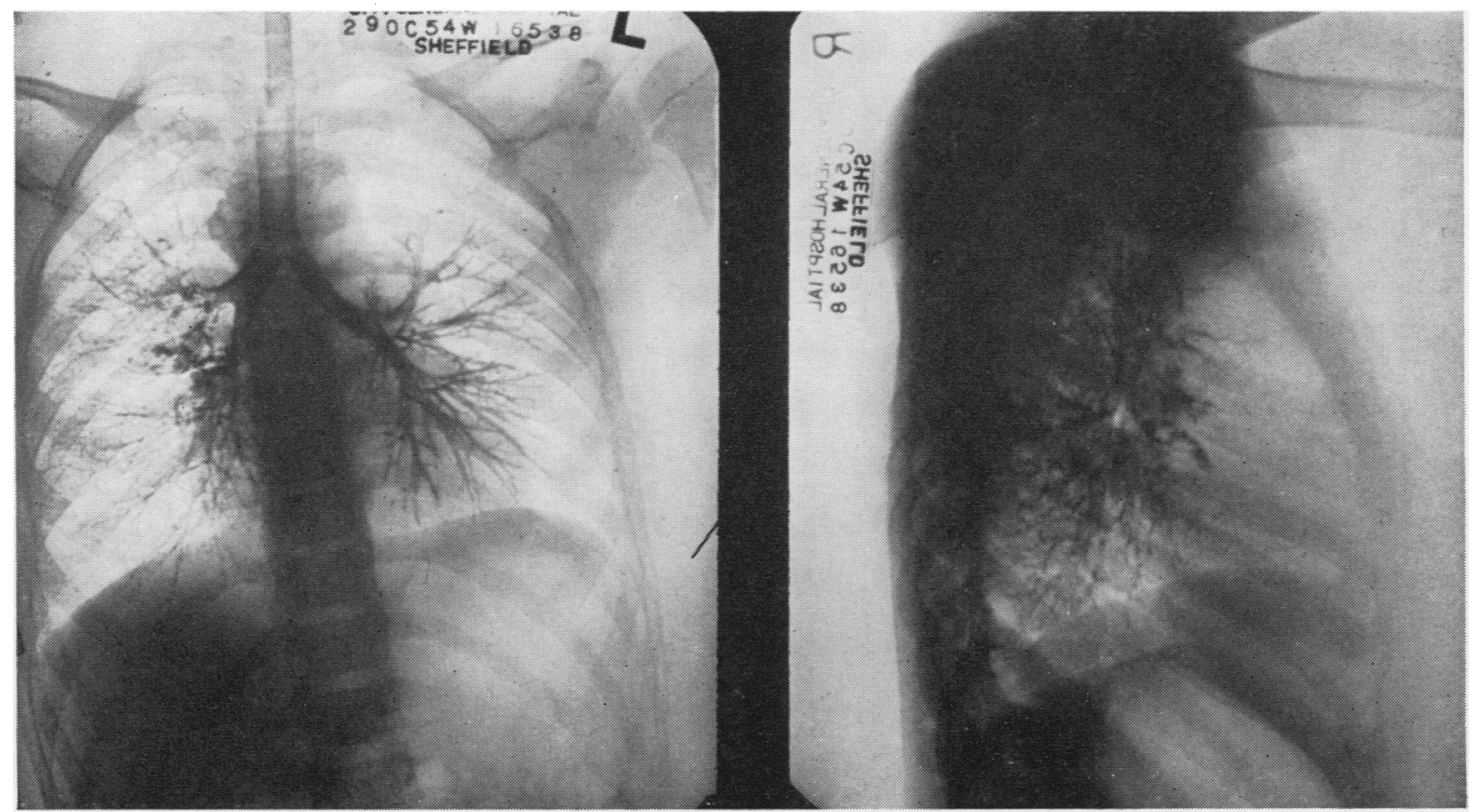

Fig. $4 a$ and $b$.-Bronchogram taken six years after excision of a mass of glands in the right middle lobe region showing residual bronchiectasis. 
Fig. 5.t.-Film showing a mass and collapse of the right upper lobe. mass of caseating glands was excised, and recovery, without any antibiotic, was uneventful. A bronchogram six years later showed some dilatation in the upper lobe bronchi. This might have been avoided by an earlier operation, and though the patient is symptomless now (1957) it may give rise to trouble later.

The point of relatively early operation in these cases is shown more clearly in the next (Figs. $4 a, 4 b$ ):
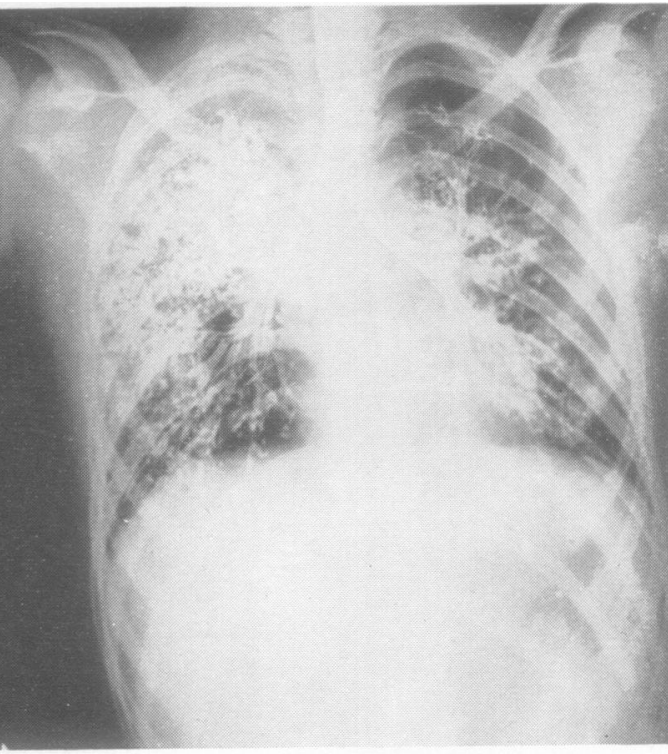

FIG. $5 b$.-Bronchogram showing outline of a paratracheal mass

CASE Note.-D. H., a girl, born in September, 1931 (Hospital No. 53/1711), came under my care in February 1942 , after 16 weeks' illness. Radiographs showed collapse of the right upper lobe and a large paratrachea $\vec{E}$ mediastinal mass. Operation on June 9 suggested tha excision was impossible, so nothing was done. She developed bronchiectasis of the right upper lobe, and 11 (1953) years later this was removed on account of

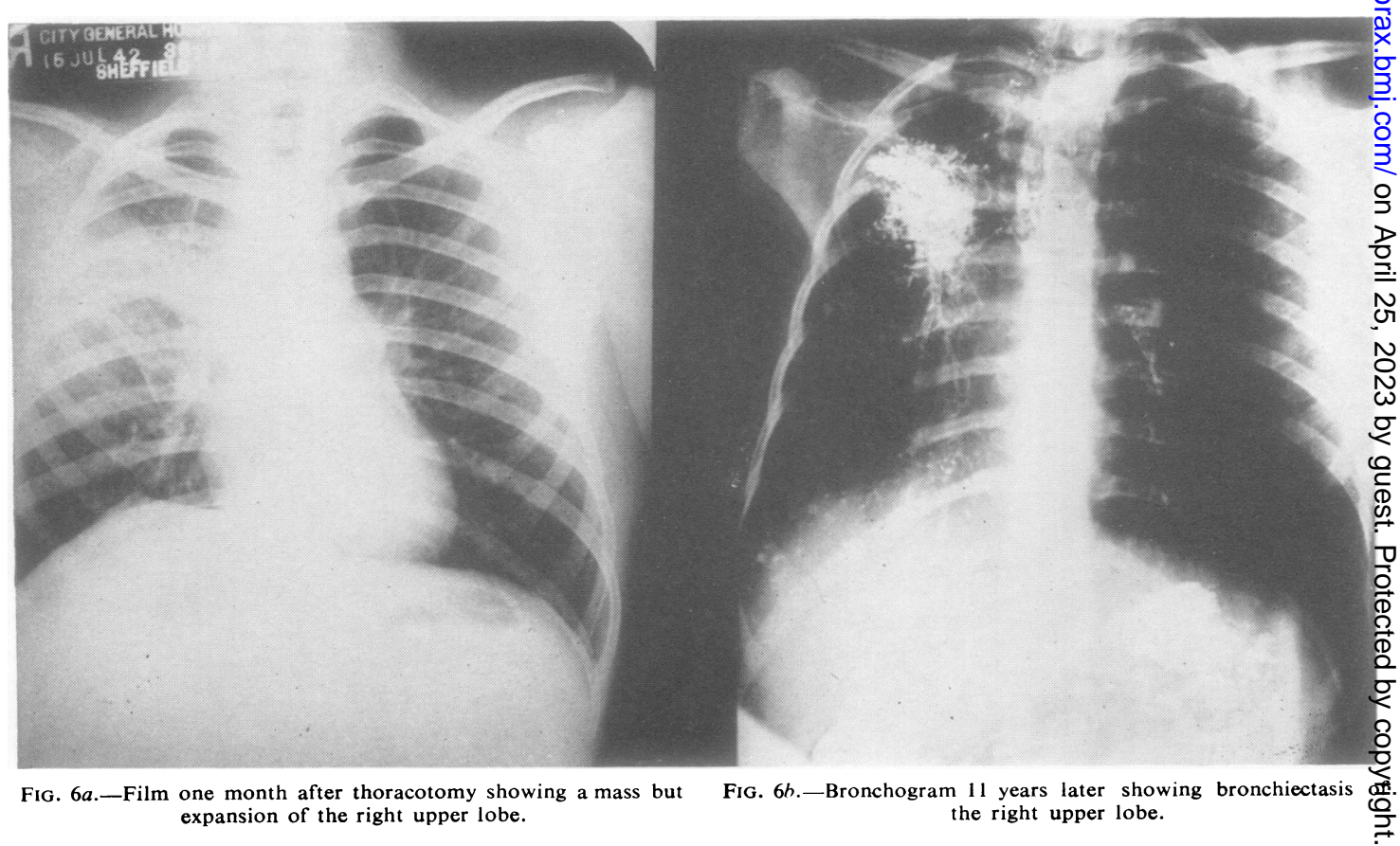




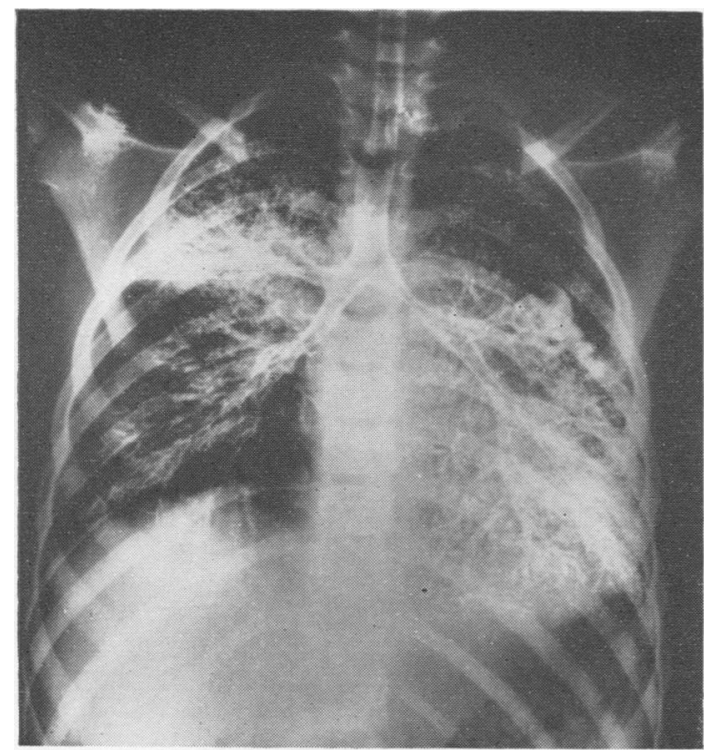

FIG. 7.-Pre-operative bronchogram showing bronchiectasis of the left upper lobe and stricture of the left main bronchus.

marked and increasing cough and expectoration, since when she has been well (1957).

The mistake in this patient was that the mass should have been incised and scraped out if it could not be excised (Figs. $5 a, 5 b, 6 a, 6 b$ ).

4. Glandular pressure which gives rise to fibrous bronchial stenosis. This requires one of three procedures:

(a) Resection of stricture plus pulmonary tissue for total destruction of the latter. (b) Resection of stricture plus anastomosis if pulmonary tissue distal to the stricture is capable of function. (c) Resection of stricture plus anastomosis with excision of diseased area and conservation of functional areas of lung tissue. The anastomosis may be of bronchus to bronchus or of bronchus to carina.

The third procedure is thus illustrated:

CASE NOTE.-R. D., a girl, was born on January 28, 1947 (Hospital No. 55/45811). In 1948 this patient was under treatment for tuberculous meningitis from which she made a satisfactory recovery. Since 1953 she has suffered from recurrent episodes of cough and expectoration. A bronchogram (1955) showed a stenosis of the left main bronchus and bronchiectasis of the left upper lobe. On $N_{1}$ arch 11,1955 , the left upper lobe was removed and the stricture excised with anastomosis of

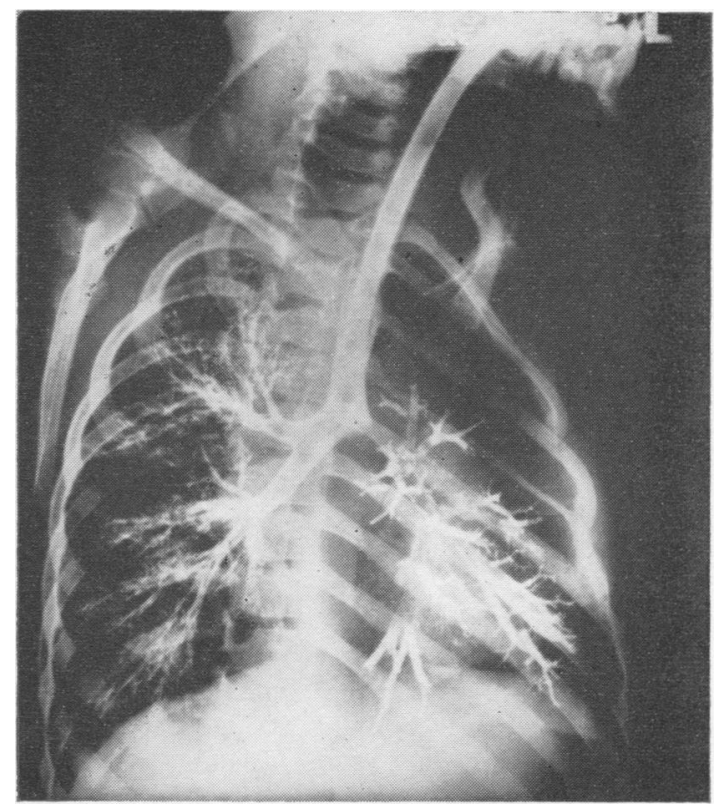

FIG. 8.-Post-operative bronchogram after resection of the left upper lobe, excision of the bronchial stricture, and anastomosis of the left lower lobe bronchus to the carina.

the left lower lobe bronchus to the carina. The result (1957) has been satisfactory (Figs. 7, 8).

5. The residual fibrosis of the gland mass may lead to superior vena caval obstruction or subcarinal oesophageal obstruction.

The latter may require a careful lysis operation. but the results of resection and graft for this type of vena caval obstruction are poor as there is associated thrombosis as well as fibrotic stricture and kinking. Even the use of the patient's common iliac vein has been rapidly followed by thrombosis.

\section{SUMMARY}

The indications for surgical intervention and the operative procedures required in the primary pulmonary tuberculous complex of childhood are discussed.

It is a pleasure to record thanks to my colleasues, Dr. E. K. Abbott, consultant radiologist, and Dr. J. Johnston, consultant anaesthetist, for their long. suffering.

\section{REFERENCES}

Bentley, F. J., Grzybowski, S., and Benjamin, B. (1954). Tuberculosis in Childhood and Adolescence. NAPT, London.

Lorber, J. (1957). Personal communication. 